\title{
Association between Capnogram and Respiratory Flow Rate Waveforms during Invasive Mechanical Ventilation
}

\author{
C. C. Rasera and P. M. Gewehr
}

\begin{abstract}
Capnography refers to the continuous and noninvasive measurement of carbon dioxide $\left(\mathrm{CO}_{2}\right)$ concentration in respiratory gases and it provides a real-time assessment of ventilatory status. The aim of this paper is to demarcate the inspiratory and expiratory segments of a time capnogram using data from respiratory flow rate waveforms. The measurements were obtained from 38 infants under mechanical ventilation in intensive care unit. A comparison was made between $\mathrm{CO}_{2}$ and respiratory flow waveforms to determine the inspiratory segment (phase 0 ) and the expiratory segment and its subdivisions (phases I, II, and III). The coefficients of determination were $0.83(p<0.001)$ for end-tidal $\mathrm{CO}_{2}$ pressure and inspiratory flow during rebreathing; and $0.97(p<0.001)$ during the weaning process. The end of expiration almost coincides with the downslope of the $\mathrm{CO}_{2}$ waveform in the capnograms when there is no rebreathing, during the weaning process. However, in the presence of rebreathing, the alveolar plateau is prolonged and includes a part of inspiration, in addition to the expiratory alveolar plateau.
\end{abstract}

Index Terms-Capnogram, invasive mechanical ventilation, monitoring device, respiratory flow rate.

\section{INTRODUCTION}

Capnography refers to the continuous and noninvasive measurement of carbon dioxide $\left(\mathrm{CO}_{2}\right)$ concentration in respiratory gases, which is often by infrared made spectrometric analysis during the respiratory cycle. All capnographs provide a single value for $\mathrm{CO}_{2}$, usually from end-respiration to end-respiration, designated end-tidal $\mathrm{CO}_{2}$ pressure $\left(\mathrm{PetCO}_{2}\right)$ and a continuous plot of expired $\mathrm{CO}_{2}$ levels over time, producing a capnogram [1], [2].

The information provided by the capnography, as $\mathrm{PetCO}_{2}$ and the waveform capnogram can be used as a tool to pulmonary problem diagnoses and respiratory monitoring during the time that the patient remains in invasive mechanical ventilation (IMV) [3], [4]. The device can also be used to monitor patients in emergency response situations, during anesthesia procedure and pediatric intensive care unit [5].

The capnogram shows breath-to-breath $\mathrm{CO}_{2}$ elimination curves, and it allows visual inspection of changes in $\mathrm{CO}_{2}$ concentrations by means of a waveform on the screen, paper recording, or even digitized measurements [2]. In addition,

Manuscript received October 15, 2012; revised January 2, 2013. This work was supported in part by means of a PhD. scholarship from Capes Agency (Brazilian Ministry of Education).

The authors are with the Biomedical Engineering Programme, Parana Federal University of Technology (UTFPR), and the Pediatric Intensive Care Unit of Pequeno Principe Hospital, Parana, Brazil (e-mail: carmen_enge@yahoo.com.br, gewehrs@utfpr.edu.br). the capnography provides a real-time assessment of ventilatory status $[6]$.

The usual representation of capnograms that is commonly used in clinical practice is $\mathrm{CO}_{2}$ concentration displayed against time during inspiration and expiration, with $\mathrm{PetCO}_{2}$ on the $\mathrm{Y}$-axis and time on the $\mathrm{X}$-axis. The shape of the capnogram usually has four phases and two angles (Fig. 1) [1], [7].

The normal adult capnogram has an almost square wave pattern (Fig. 1), marked by alternating inspiratory (phase 0 ) and expiratory phases. Expiration itself consists of three successive phases: (I) a latency phase, corresponding to the expiration of the anatomical dead space, thus the $\mathrm{CO}_{2}$ concentration equals zero, because the dead space is void of $\mathrm{CO}_{2}$; (II) slope phase, marked by an increase abruptly of $\mathrm{CO}_{2}$ concentration, corresponding to expiration of mixed air; and (III) plateau phase, reflecting the elimination of alveolar air resulting in a peak at the end of tidal expiration $\left(\mathrm{PetCO}_{2}\right.$ close to alveolar carbon dioxide tensions) [2], [8].

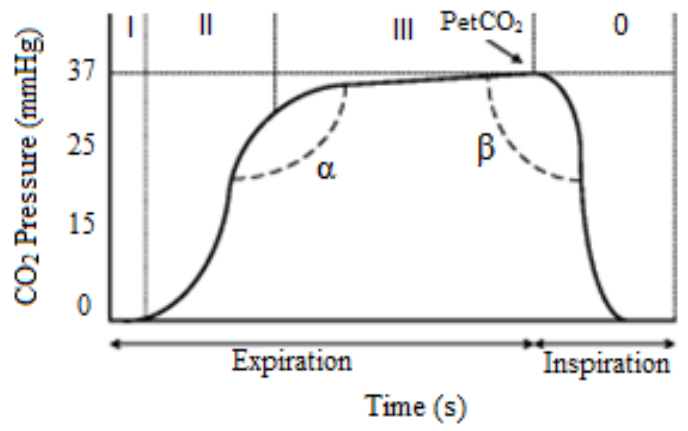

Fig. 1. Example of a normal capnogram with the inspiratory (phase 0) and expiratory phases (I, II, and III), $\alpha$ and $\beta$ angles and end-tidal carbon dioxide pressure $\left(\mathrm{PetCO}_{2}\right)$

These three phases are separated by two well-defined transitions: $\alpha$ and $\beta$ angles [3]. In normal conditions, the alpha angle is approximately $110^{\circ}$, this angle can increase in certain situations (e.g. in patients with altered ventilation-perfusion). The second angle in the capnogram (beta) occurs between phase III and the beginning of the inspiration. In normal conditions, the beta angle is nearly 90 degrees; however, it can increase in situations where $\mathrm{CO}_{2}$ is reinhaled [1], [9].

Partial reinhalation of previously exhaled $\mathrm{CO}_{2}$ may impair the efficacy of mechanical ventilation in improving $\mathrm{CO}_{2}$ removal and unloading ventilatory muscles. At constant alveolar ventilation, any $\mathrm{CO}_{2}$ concentration above zero in inhaled gases causes an increase of arterial $\mathrm{CO}_{2}$ tension by an equal amount. Accordingly, significant $\mathrm{CO}_{2}$ rebreathing increases alveolar ventilation requirements to maintain desired arterial $\mathrm{CO}_{2}$ tension. This can limit the beneficial effects of inspiratory assistance provided by IMV [10]. 
According to Thomas et al. [7] the review of the capnograph waveform may help the physician in diagnosis and establishing response to treatment in patients with respiratory alteration and other conditions. In addition, to being able to recognize the normal shape of the capnogram, physicians need to be familiar with the most common abnormal shapes that can occur and with the capnogram segments [1].

Thus, the aim of this paper is to demonstrate the advantage of demarcating the inspiratory and expiratory segments of a time capnogram using the data from expiratory and inspiratory flow rate waveforms. In addition, delineating various components of a time capnogram would allow us to better understand the components of normal and pathological waveforms.

\section{MATERIAL AND METHODS}

This study was approved by the ethics committee of Pequeno Principe Hospital, Curitiba, Brazil. The research was carried out in the hospital's cardiac critical care unit between September 2011 and January 2012. Informed consent was obtained from the parents or the caregivers responsible for the patients.

A total of 38 infants were evaluated from 2 days to 3 months of age, both sexes during the post-operative period of cardiac surgery. The characteristics of the patients are presented in Table I. Patients who had sepsis, some respiratory complication (e.g. pneumonia and pleural effusion) or the children who progressed to death were excluded. Vital parameters and ventilator settings were continuously monitored.

The capnographic curves and respiratory flow rates were analyzed in two moments during the period that the patient remained in intensive care unit:

A) During the use of mechanical ventilation equipment, at the time that the physician suspected of rebreathing;

B) During weaning process, before extubation of IMV, in this stage the patient was in good clinical conditions.

\section{TABLE I: CHARACTERISTICS OF THE PATIENT.}

\begin{tabular}{lcc}
\hline \hline Characteristic & \multicolumn{2}{c}{ Patient $(n=38)$} \\
\hline Age (month) & $1.41 \pm 0.6$ \\
Sex (male/female) & \multicolumn{2}{c}{$15 / 23$} \\
Weight $(\mathrm{kg})$ & \multicolumn{2}{c}{$2.30 \pm 1.6$} \\
Vital Parameters & Instant A & Instant B \\
Cardiac Frequency & $79.7 \pm 3.5$ & $80.1 \pm 1.5$ \\
Respiratory Frequency & $27 \pm 2.8$ & $18 \pm 2.2$ \\
Body temperature $\left({ }^{\circ} \mathrm{C}\right)$ & $36.4 \pm 1.2$ & $36.7 \pm 1.2$ \\
Pulse Oxygen Saturation $(\%)$ & $96.1 \pm 1.7$ & $99.3 \pm 1.1$ \\
\hline \hline \multicolumn{2}{l}{ Values are expressed as mean \pm standard deviation or number of patients. }
\end{tabular}

Patients undergoing IMV were ventilated by Inter 5 Ventilator (Intermed, São Paulo, Brazil), using limited pressure, time-cycled ventilators in either assist control mode or synchronous intermittent mandatory ventilation mode.

$\mathrm{PetCO}_{2}$ and $\mathrm{CO}_{2}$ waveforms were monitored using a sidestream capnography module (Fig. 2) ( $\mathrm{CO}_{2} \mathrm{CGM}$ OA1000, Ronseda, Shenzhen, China) placed between endotracheal tube and the circuit of ventilator through an airway adapter. The $\mathrm{CO}_{2}$ monitoring began immediately after calibration performed according to the manufacturer's recommendations.

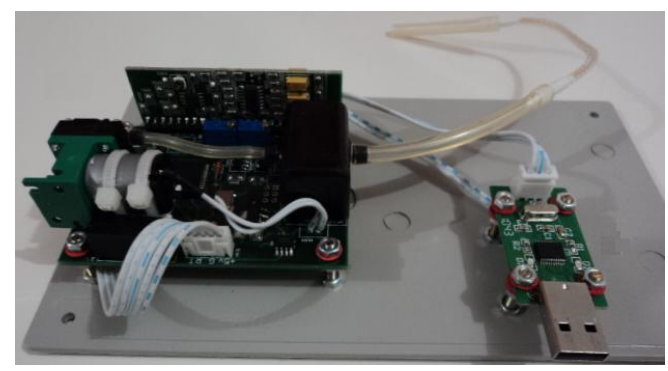

Fig. 2. Sidestream capnography module.

The data of respiratory flow rates were obtained from GMX Slim graphic monitor (Intermed, SP, Brazil) which was coupled to the mechanical ventilation. The analysis was done through an adapter connected between the endotracheal tube and the ventilator circuit. The monitor data were saved through Wintracer Software (Version 3.3.5 Beta, Intermed, SP, Brazil) in a notebook.

In order to measure the deformation of capnogram related to time; some variations of waveform parameter were calculated; they were defined in relation to the beginning of expiration $\left(\mathrm{T}_{0}\right)$, i.e. from the start of phase II on the capnographic curve.

On each of the recordings, the beginning and the end of inspiration and expiration were identified on the flow rate waveforms. Perpendicular lines were drawn through these points to intercept $\mathrm{CO}_{2}$ waveforms to determine the inspiratory and expiratory segments of each capnogram in the study.

In addition, two indices were measured indirectly:

The $\alpha$ angle between the intermediate and the initial slopes;

The $\beta$ angle was defined by the prolongation of the line between the terminal slope (phase III) and the descending slope (phase 0).

For comparison and quantitative analysis of $\mathrm{CO}_{2}$ waveforms in two moments of each patient, it was necessary to select good quality cycles according to criteria of amplitude, duration and, when possible, regularity of the curve [11].

In this study, we systematically eliminated the $\mathrm{CO}_{2}$ cycles that did not meet the following criteria: 1) validity lasting between 1 and 4 seconds; 2) symmetry for at least 5 cycles; and 3) good regularity of expiratory phases.

The data recorded were analyzed statistically; the association between $\mathrm{PetCO}_{2}$ and respiratory flow rate was analyzed using the Pearson product-moment correlation coefficient and simple linear regression. Statistical analysis was performed using MedCalc Statistical Software version 10.4.5 (Mariakerke, Belgium).

\section{RESULTS}

The waveform analysis was assessed during the use of IMV, at the time that the physician suspected of rebreathing. For the 38 measurements, $\mathrm{PetCO}_{2}$ was $37.1 \pm 1.28 \mathrm{mmHg}$ 
and inspiratory flow value was $7.51 \pm 0.8 \mathrm{~L} / \mathrm{min}$. The correlation between flow rate and $\mathrm{PetCO}_{2}$ measurements was $r^{2}=0.8336(\mathrm{p}<0.001)$ (Fig. $\left.3 \mathrm{~A}\right)$.

During the weaning process, before extubation of IMV, the $\mathrm{PetCO}_{2}$ mean value was $39.6 \pm 1.35 \mathrm{mmHg}$, while the inspiratory flow value was $6.28 \pm 0.9 \mathrm{~L} / \mathrm{min}$. The correlation between flow rate and $\mathrm{PetCO}_{2}$ measurements was $r^{2}=0.9744$ $(p<0.001)$ throughout the study period (Fig. $3 \mathrm{~B})$.

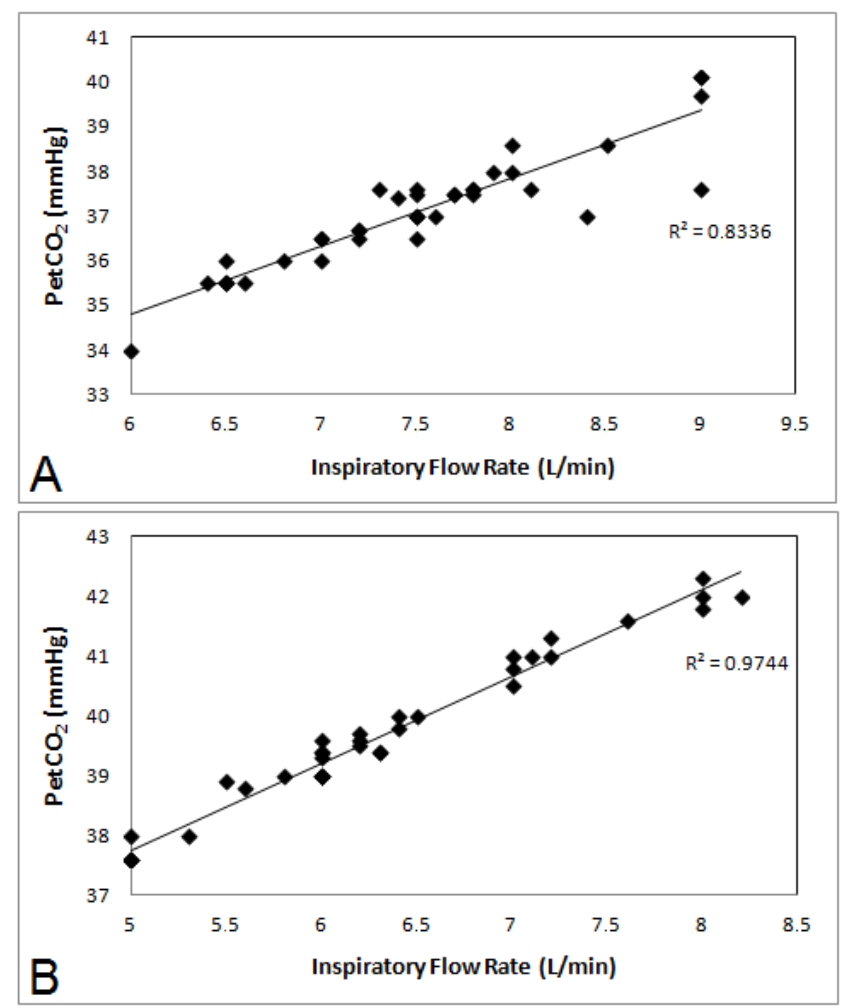

Fig. 3. The correlation between the end-tidal carbon dioxide pressure $\left(\mathrm{PetCO}_{2}\right)$ and the inspiratory flow rate. A - during prolonged mechanical ventilation; and $\mathrm{B}$ - during weaning process from ventilatory assistance.

The mean period that the patients remained in IMV was 14 days, the minimum and maximum time of IMV were 11 and 19 days, respectively.

When capnogram angles were analyzed, the patients had a mean value of $\alpha$ angle of $115^{\circ} \pm 1.3^{\circ}$ and $\beta$ angle of $90.4^{\circ} \pm$ $1.8^{\circ}$, during weaning process. However, in capnograms recorded in patients with rebreathing the mean value for $\alpha$ and $\beta$ angles were $117^{\circ} \pm 2.1^{\circ}$ and $106.4^{\circ} \pm 3.23^{\circ}$, respectively, as shown by the shaded area beyond phase III with the $\beta$ angle higher than $90^{\circ}$ (Fig. 4).

Fig. 4 shows the respiratory flow rate waveform and capnogram recorded during a suspected of rebreathing with the patient under prolonged mechanical ventilation, whereas, Fig. 5 represents the measurements during the weaning process from ventilatory assistance.

The $a b$ segment represents inspiration, and the $b a$ segment represents expiration on the respiratory waveforms. These designations were used to demarcate inspiratory and expiratory segments of respective time capnograms. The expiration ended at phase III on the time capnogram. The shaded area under the $\mathrm{CO}_{2}$ curve beyond the extrapolated line represents the inspiratory phase of the respiratory cycle, thus constituting rebreathing.

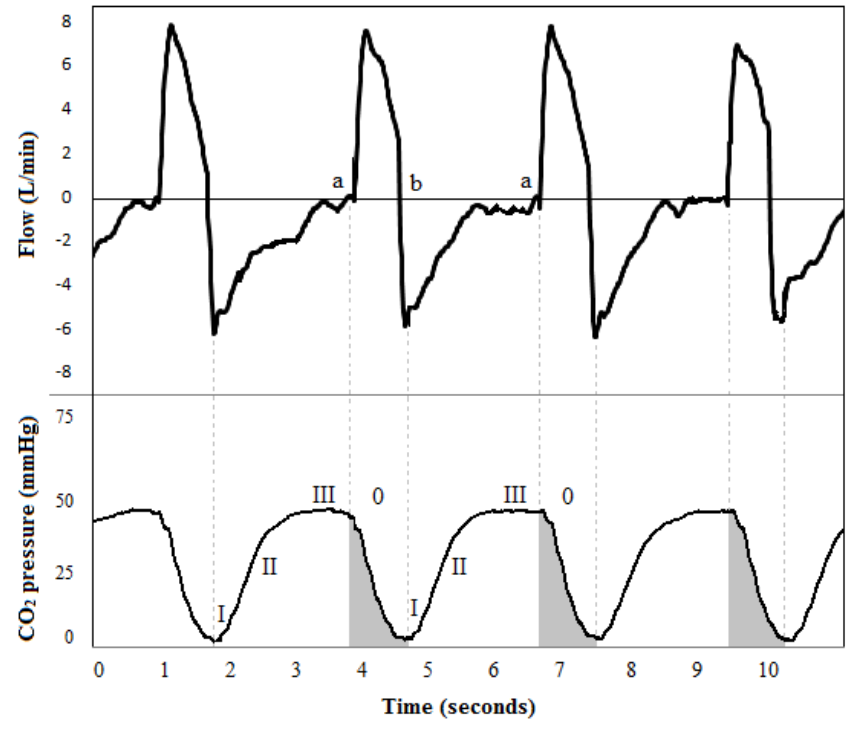

Fig. 4. Respiratory flow rate waveform and capnogram recorded during prolonged invasive mechanical ventilation with $a b=$ inspiration; $b a=$ expiration; $0=$ inspiration phase; I, II, and III = expiration phase.

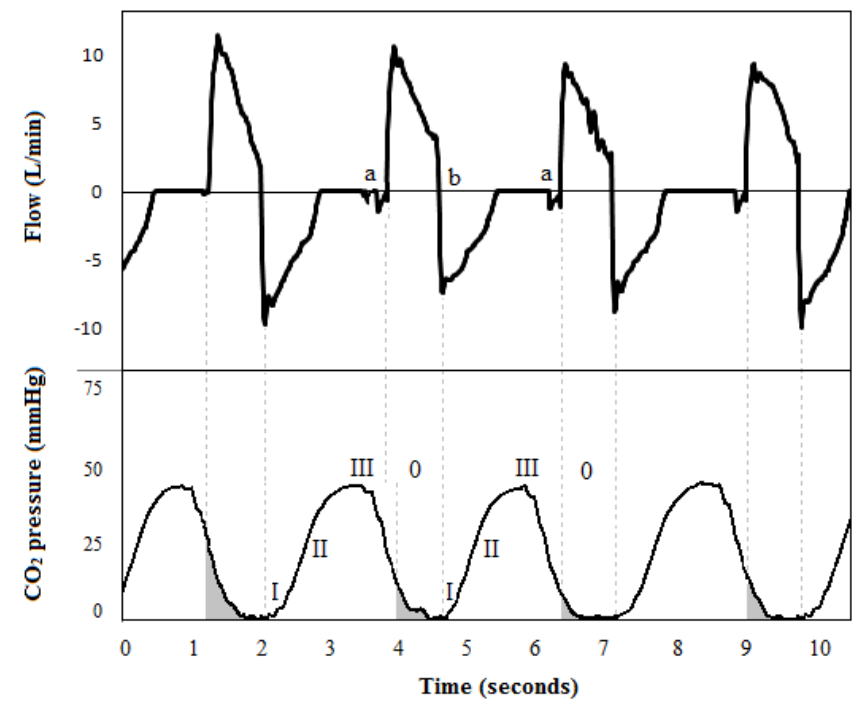

Fig. 5. Respiratory flow rate waveform and capnogram recorded during weaning process from ventilatory assistance with $a b=$ inspiration; $b a=$ expiration; $0=$ inspiration phase; I, II, and III = expiration phase.

\section{DISCUSSION}

Capnography has become the standard of care for basic respiratory monitoring for all intubated patients in the intensive care unit, and $\mathrm{PetCO}_{2}$ is commonly measured during mechanical ventilation of children in postoperative period [1]. The extension of this technique to intubated infants has been limited by technical problems associated with the capnographic indices [5], [12].

Despite the relatively simple technology behind capnography and the ease with which capnographic measurements are obtained, the interpretation of the results requires a profound knowledge of the physiological bases of $\mathrm{CO}_{2}$ production, transport, and elimination [1].

Breen and Bradley [13] suggested in 1997 that $\mathrm{CO}_{2}$ spirography $\left(\mathrm{CO}_{2}\right.$ concentrations versus inspired and expired volume during respiratory cycle) should be used to detect rebreathing. By integrating airway-measured flow and $\mathrm{CO}_{2}$ pressure, they computed overall expired and inspired $\mathrm{CO}_{2}$ 
volume during intermittent positive pressure ventilation in a circle anesthesia circuit. They concluded that $\mathrm{CO}_{2}$ spirography (in contrast to time capnography) is required to detect inspiratory valve incompetence during mechanical ventilation. However, according to Shankar and Philip [14] the method suggested by Breen and Bradley is complex and may not be suitable for routine clinical use.

Otherwise, the comparison between respiratory flow rate and $\mathrm{CO}_{2}$ waveforms to determine inspiratory and expiratory segments of the time capnogram can be easily analyzed in infants patients with instruments available in intensive care unit.

Graphic extrapolation from the respiratory flow waveform to the time capnogram clearly demarcates the inspiratory segment (phase 0) and the beginning of the expiratory segment in the time capnogram. The end of expiration almost coincides with the down slope of the $\mathrm{CO}_{2}$ waveform, there is no rebreathing or minimal rebreathing in clinically stable patients during weaning process $(\beta$ angle is approximately $90^{\circ}$ ). However, in capnograms recorded in patients with suspected rebreathing, the diagnosis was confirmed, as shown by the shaded area beyond phase III with the $\beta$ angle higher than $90^{\circ}$ (Fig. 4).

The normal adult capnogram has an almost square wave pattern, however in 2005 Thompson and Jaffe [3] described the $\mathrm{CO}_{2}$ waveform in neonatal and infants patients more rounded than the adult waveform, in accordance with Fig. 4 found in our study.

According to Shankar and Philip [14] capnographs using sidestream sensor technology may not allow comparison between $\mathrm{CO}_{2}$ waveforms and flow rate waveforms because of the longer response time of sidestream $\mathrm{CO}_{2}$ analyzers. However, we have used a sidestream capnography and this choice has not compromised the measurements and analyzes.

The analysis of $\mathrm{CO}_{2}$ pressure through capnography during prolonged mechanical ventilation of neonates is poorly documented in the pediatric literature. The additional dead space, mechanical problems, low weight, small flow and respiratory pressure may limit the clinical value of capnography with infants [3].

In order to reduce these limitations we have used the sidestream capnograph that requires a small sample cell and, therefore, a low flow rate $(50 \mathrm{ml} / \mathrm{min})$. For the neonate with high respiratory rates and low tidal volumes, this rate of gas avoids the dilution of alveolar $\mathrm{CO}_{2}$. Thus, the device provides precise measurements in newborns patients.

One of the limitations of this terminology is that it is unknown precisely where in the time-capnogram, inspiration, and expiration begin. It is generally assumed that inspiration begins when the $\mathrm{CO}_{2}$ concentration decreases abruptly at the end of phase III caused by inhalation of $\mathrm{CO}_{2}$-free gases [15]. However, this is not always true, as can be seen from our observations. The alveolar plateau is prolonged during rebreathing and the time capnograph cannot help us identify the inspiratory prolongation of the alveolar plateau occurring because of rebreathing of $\mathrm{CO}_{2}$. Thus, during rebreathing, the plateau may indeed include a part of inspiration (phase 0 ) in addition to the expiratory alveolar plateau [14].

\section{CONCLUSION}

We have analyzed $\mathrm{CO}_{2}$ concentration and respiratory flow rates from our subjects and the comparison between these two methods can determine inspiratory and expiratory segments of the time capnogram.

We believe that incorporation of flow direction information into sidestream capnography would allow a more physiologically meaningful interpretation of time capnograms. The present results could be a guideline for clinicians in the physiological interpretation of the capnogram and it could help clinicians to get accurate respiratory information about the infant patient during the rebreathing.

Demarcation of a time capnogram into inspiratory and expiratory components using flow rates will not only facilitate prompt detection of rebreathing, but will also allow application of standardized and physiologically appropriate nomenclature for better understanding and interpretation of time capnograms.

In addition, the knowledge of alteration in the $\mathrm{CO}_{2}$ waveform can help the health professionals to change the mechanical ventilatory parameters in order to obtain a capnographic wave closest to normal thereby improving the lung function of patients.

\section{ACKNOWLEDGMENT}

The authors would like to thank the team of Pequeno Principe Hospital for the support and assistance.

\section{REFERENCES}

[1] C. Domingo, L. Blanch, G. Murias, and M. Luján, "State-of-the-art sensor technology in Spain: Invasive and non-Invasive techniques for monitoring respiratory variables," Sensors, vol. 10, pp. 4655-4674, 2010 .

[2] T. A. Howe, K. Jaalam, R. Ahmad, C. K. Sheng, and N. H. N. A. Rahman, "The use of end-tidal capnography to monitor non-intubated patients presenting with acute exacerbation of asthma in the emergency department," The Journal of Emergency Medicine, vol. 41, pp. 581-589, 2009.

[7] J. E. Thompson and M. B. Jaffe, "Capnographic waveforms in the mechanically ventilated patient," Respir Care, vol. 50, pp. 100-108, 2005.

[8] T. K. Roy and J. Den Buij, "Calculating the effect of altered respiratory parameters on capnographic indices," IFMBE Proceedings, vol. 14, pp. 123-126, 2007.

[9] M. Langhan, "Continuous end-tidal carbon dioxide monitoring in pediatric intensive care unit," J. Crit. Care, vol. 24, pp. 227-230, 2009.

[10] B. Krauss, A. Deykin, A. Lam, J. J. Ryoo, D. R. Hampton, P. W. Schmitt, and J. L. Falk, "Capnogram shape in obstructive lung disease," Anesth. Analg., vol. 100, pp. 884-888, 2005.

[11] A. N. Thomas, D. J. R. Harvey, and T. Hurst, "Standards and guidlines for capnography in critical care," The Intensive Care Society, pp. 1-32, 2009.

[12] N. A. R. N. Hisamuddin, A. Rashidi, K. S. Chew, J. Kamaruddin, Z. Idzwan, and A. H. Teo, "Correlations between capnographic waveforms and peak flow meter measurement in emergency department management of asthma," Int. J. Emerg. Med., vol. 2, pp. 83-89, 2009

[13] M. R. Hawkins and N. Gilboy, "Noninvasive monitoring of end-tidal carbon dioxide in the emergency department," Advanced Emergency Nursing Journal, vol. 28, pp. 301-313, 2006.

[14] F. Mojoli and A. Braschi, "Carbon dioxide rebreathing during noninvasive mechanical ventilation," Noninvasive Mechanical Ventilation, vol. 7, pp. 77-82, 2012.

[15] B. You, R. Peslin, C. Duvivier, V. Dang Vu, and J. P. Grilliat, "Expiratory capnography in asthma," Eur Respir J, vol. 7, pp. 318-323, 1994. 
[16] J. J. Hagerty, M. E. Kleinman, D. Zurakowski, A. C. Lyons, and B. Kraus, "Accuracy of a new low-flow sidestream capnography technology in newborns," Journal of Perinatology, vol. 22, pp. 219-225, 2002.

[17] P. H. Breen and P. J. Bradley, "Carbon dioxide spirogram (but not capnogram) detects leaking inspiratory valve in a circle circuit," Anesth. Analg, vol. 85, pp. 1372-1376, 1997.

[18] K. B. Shankar and J. H. Philip, "Defining segments and phases of a time capnogram," Anesth. Analg, vol. 91, pp. 973-977, 2000.

[19] I. M. Cheifetz and T. R. Myers, "Should every mechanically ventilated patient be monitored with capnography from intubation to extubation?" Respir. Care, vol. 52, pp. 423-442, 2007.

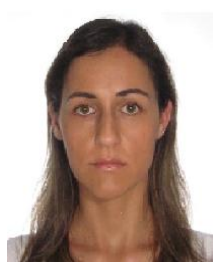

Carmen Caroline Rasera was born in Parana, Brazil, 1984. She graduated in Physical Therapy from the Evangelical College of Parana (2005), improvement in Pediatric Physical Therapy by the Pequeno Principe Hospital (2006), specialization in Cardiopulmonary Physiotherapy from Excellence Centre in Postgraduate in Health (2007) and M.Sc. in Biomedical Engineering from the Federal Technological University of Parana
(2010). She is currently a Ph.D. student in Biomedical Engineering from Federal Technological University of Parana, exclusive dedication. Her research interests include the following topics: respiratory parameters measurement, invasive mechanical ventilation and pediatrics.

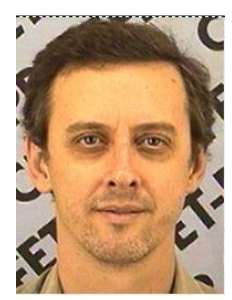

Pedro Gewehr was born in Parana, Brazil, 1957. He graduated in Electrical Engineering from Federa University of Parana (1980), undergraduate in electronics for the full degree level by the Federal Technological University of Parana (1985), M.Sc. in Electrical Engineering from Estadual University of Campinas (1982) and Ph.D. in Medical Physics Bioengineering from the University of London (1991) $\mathrm{He}$ is currently a professor of higher education in Federal Technological University of Parana (Brazil) and he has experience in the area of Biomedical Engineering. His research interests include the following topics: optical oxygen sensor, lifetime phosphorescence quenching, metalloporphyrin probe. 